\title{
Mitteilungen der DRL
}

Z Rheumatol 2012 $\cdot 71: 440$

DOI 10.1007s00393-012-1033-5

(c) Springer-Verlag 2012
Herausgegeben im Auftrag des Vorstandes

Prof. Dr. Erika Gromnica-Ihle Präsidentin Deutsche Rheuma-Liga Bundesverband e.V. Maximilianstr. 14

53111 Bonn

Tel.: 0228-76606-0 bv@rheuma-liga.de www.rheuma-liga.de
Redaktion

Susanne Walia

Referentin für Öffentlichkeitsarbeit

Deutsche Rheuma-Liga

Bundesverband e.V.

53111 Bonn

Maximilianstr. 14

Tel.: 0228-76606-11

bv.walia@rheuma-liga.de

www.rheuma-liga.de
Bundesverband e.V.

\section{Aktiv Tour startet durch Deutschland}

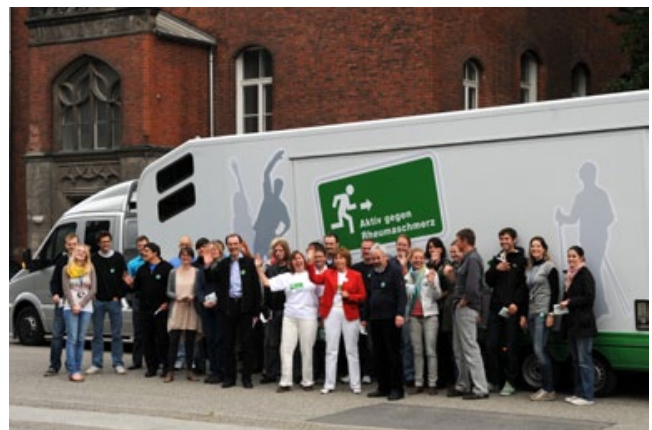

Truck-Stopp im Deutschen Rheumaforschungszentrum

Am Mittwoch, den 6. Juni 2012, eröffnete Bundesgesundheitsminister Daniel Bahr die Aktiv-Tour 2012 auf dem Potsdamer Platz in Berlin. Als Schirmherr der Kampagne „Aktiv gegen Rheumaschmerz" würdigte der Minister die Arbeit der Deutschen Rheuma-Liga: „Mit ihrem weitreichenden Angebot an Beratung, Bewegung und Erfahrungsaustausch leistet die Rheu-

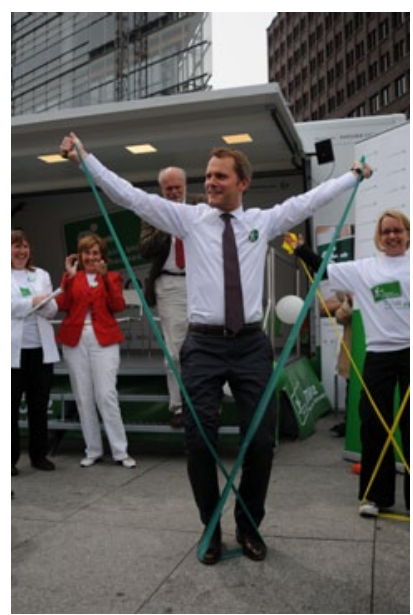

Bundesgesundheitsminister eröffnet den Bewegungsparcours
ma-Liga für das Gesundheitswesen einen sehr wertvollen Beitrag. Die Arbeit der zahlreichen ehrenamtlichen und engagierten Mitglieder ist sehr wichtig für die Patientinnen und Patienten und eine sehr sinnvolle Ergänzung der Arbeit von Ärzten und Krankenkassen." Der Minister betonte, dass die Aktiv-Tour ganz besonders unterstützenswert sei, da sie die im Alltag notwendige Bewegung in den Mittelpunkt stelle. Minister Bahr ging mit gutem Beispiel voran und eröffnete gemeinsam mit Gudrun Baseler, Bundesjugendsprecherin der Deutschen Rheuma-Liga, den Bewegungsparcours.

In ihrem Grußwort verwies Präsidentin Prof. Gromnica-Ihle auf den zeitgleich stattfindenden EULAR Kongress in Berlin und betonte, dass Rheuma nicht mehr nur als Krankheit alter Menschen begriffen werden dürfe: Sehr viele der 20 Millionen Betroffenen in Deutschland seien junge $\mathrm{Pa}$ tienten. Als wichtigste Therapie neben der medikamentösen Behandlung stellte sie die Bewe-

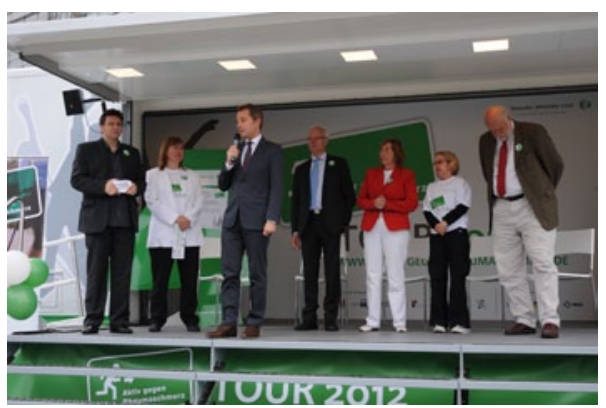

Talk-Runde auf der Bühne

gungstherapie heraus, die den Kernpunkt der Aktiv-Tour bildet. Prof. Gromnica-Ihle forderte, dass es auch zu mehr Bewegung innerhalb der Politik kommen müsse. So müssten beispielsweise die Patientenrechte bei fehlerhaften Behandlungen deutlich gestärkt werden. Dabei versprach Sie, dass die Deutsche RheumaLiga sich weiterhin einmischen werde, um solche Themen gesellschaftlich in Bewegung zu bringen.

Am Talk auf der Bühne beteiligten sich auch Prof. Jürgen Braun, Präsident der Deutschen Gesellschaft für Rheumatologie, sowie Prof. Uwe Niethard, Generalsekretär der Deutschen Gesellschaft für Orthopädie und Orthopädische Chirurgie. Niethard sprach sich für die tägliche Bewegung als ein Muss für mehr Mobilität im Alter aus: „Tausend Schritte täglich“, seien ein guter Anfang. Prof. Jürgen Braun würdigte den europäischen Kongress EULAR 2012: Dieser habe sich über die Jahre hinweg als gleichwertiger Kongress zum amerikanischen Rheumatologen-Kongress etabliert. Als großen Schritt in der Medikamentenforschung stellte Prof. Braun den Einsatz von Biologika her- aus, die in ihrer Bedeutung der Entdeckung der Kortikosteroide für rheumatische Erkrankungen gleichkäme.

Im Laufe der Veranstaltung protestierten Aktivisten aus Thüringen, Berlin und Brandenburg auf dem Potsdamer Platz gegen die fortschreitende Schließung von Therapiebädern, ohne die viele Bewegungsangebote der Rheuma-Ligen nicht mehr stattfinden können. In ganz Deutschland sind in den letzten Jahren Therapiebäder geschlossen worden. „In Berlin standen vor 10 Jahren 70 Therapiebäder offen, heute sind es noch 48 ", informierte Dr. Helmut Sörensen, Präsident der Deutschen Rheuma-Liga Berlin in der Gesprächsrunde mit der Staatssekretärin für $\mathrm{Ge}$ sundheit in Berlin, Emine Demirbüken-Wegner und dem Gesundheitspolitischen Sprecher der SPD, Thomas Isenberg.

Nach zahlreichen Stationen im Juni wird der Aktiv-Truck seine Deutschland-Tour im September fortsetzen.

Weitere Informationen, Bilder und ein Video zur Eröffnung im Internet:

www.aktiv-gegenrheumaschmerz.de 